\title{
Short communication: Occurrence of Arcobacter species in industrial dairy plants
}

\author{
A. Serraino and F. Giacometti ${ }^{1}$ \\ Department of Veterinary Medical Sciences, Via Tolara di Sopra 50, 40064 Ozzano Emilia (BO), Italy
}

\begin{abstract}
The present study investigated the presence of Arcobacter spp. in industrial dairy plants. Between February and September 2013, pasteurized milk used for cheesemaking, processing and cleaning water, cheese, and environmental samples from different plant sites, including surfaces in contact or not in contact with food, were sampled. A total of 126 samples were analyzed by the cultural method and isolates were identified by multiplex PCR. Arcobacter spp. were isolated from 22 of 75 environmental samples (29.3\%): of them, $22.7 \%$ were surfaces in contact with food and $38.7 \%$ surfaces not in contact with food. A total of 135 Arcobacter spp. isolates were obtained; of these, 129 and 6 were identified as Arcobacter butzleri and Arcobacter cryaerophilus, respectively. All food processing water and pasteurized milk samples were negative for Arcobacter species. We were not able to determine the primary source of contamination, but the isolation of both $A$. butzleri and A. cryaerophilus in surfaces in contact with food before and during manufacturing suggests that Arcobacter spp. are not or are only partially affected by routine sanitizing procedures in the industrial dairy plants studied. The efficacy of sanitizing procedures should be evaluated and further studies are needed to determine whether certain Arcobacter strains persist for long periods of time in industrial dairy plants and whether they can survive in different types of cheese in cases of postprocessing contamination.
\end{abstract}

Key words: Arcobacter butzleri, Arcobacter cryaerophilus, dairy plant, cheese

\section{Short Communication}

The Arcobacter genus has been linked to animal and human illness (Ho et al., 2006). In particular, the species Arcobacter butzleri and Arcobacter cryaerophilus have been associated with several cases of gastrointestinal disease, with persistent diarrhea as the main

Received November 5, 2013

Accepted December 30, 2013.

${ }^{1}$ Corresponding author: federica.giacometti3@unibo.it symptom in humans (Collado and Figueras, 2011). A direct connection between consumption of Arcobactercontaminated food and water and human illness has not yet been established, although it is likely that arcobacters are transmitted via these routes (González and Ferrús, 2011). Arcobacter spp. have been isolated from fecal samples of dairy animals (Piva et al., 2013; Shah et al., 2013) and found to contaminate different foods of animal origin, including milk (Ertas et al., 2010; Shah et al., 2012). Furthermore, 2 recent studies (Giacometti et al., 2013a,b) reported the first isolation of A. butzleri from environmental samples collected in an artisanal cheese factory, in a ready-to-eat cheese produced for retail, and in an industrial dairy plant. Given the scant data available to date, we investigated the occurrence of Arcobacter spp. in several industrial dairy plants.

The survey investigated the presence of Arcobacter spp. in 3 unrelated dairy plants (1 to 3 ) located in 3 different Italian regions. All dairy plants used only pasteurized cow milk for cheese production, and a high level of automation and good hygiene practices were in place. Dairy plants 1, 2, and 3 used a daily mean of $180,000,445,000$, and $120,000 \mathrm{~kg}$ of milk, respectively, per plant for cheesemaking.

Plant 1 had 2 completely separate production lines (A and B) producing, respectively, Mozzarella cheese and Stracchino cheese (a soft, creamy, unripened cheese that undergoes a short maturation period to obtain its typical texture). Plant 2 produced only Mozzarella cheese, whereas plant 3 produced Mozzarella cheese and Ricotta cheese (whey cheese). Plants 1 and 3 treated processing water by filtration and chlorination (1-1.5 ppm), whereas plant 2 treated the water by filtration, chlorination (1-1.5 ppm), and ozonization. All 3 plants performed a combination of cleaning-in-place and manual sanitation, using different sanitizing agents in different parts of the plants and production lines. Details of the sanitizing agents used are given in Table 1.

Sampling was done between February and September 2013. Plant 1 was sampled twice: in the first sampling, only the Mozzarella cheese line was considered, whereas the second sampling considered both lines (Mozzarella and Stracchino cheese lines); plants 2 and 3 were sampled once. A total of 126 samples were collected, placed 
in a Stomacher bag, and delivered to the laboratory in a portable refrigerator $\left(5 \pm 3^{\circ} \mathrm{C}\right)$. Samples included 12 samples of pasteurized milk used for cheesemaking, 12 processing water samples, 25 food samples, and 75 environmental samples; 2 additional samples were collected by swabbing toilet surfaces in plant 1 . Environmental samples were obtained by sponging an area of approximately $250 \mathrm{~cm}^{2}$, representing the maximum area that could be sampled for all sampling sites. Sponging within the area consisted of 10 vertical passes (up and down was considered 1 pass) and 10 horizontal passes (side to side was considered 1 pass) for each large side of the sponge. Details of samples collected in each dairy plant are reported in Table 2.

Samples were processed within 2 to $24 \mathrm{~h}$ of collection and isolation was performed according to Houf et al. (2001): $25 \mathrm{~mL}$ of liquid samples or $25 \mathrm{~g}$ of solid samples and sponges were placed in a Stomacher bag and homogenized for $5 \mathrm{~min}$ in a stomacher in $225 \mathrm{~mL}$ of Arcobacter broth (Oxoid Ltd., Basingstoke, UK) supplemented with $5 \%$ laked horse blood (Oxoid Ltd.) and a mix of cefoperazone $(16 \mathrm{mg} / \mathrm{L})$, amphotericin B (10 mg/L), 5-fluorouracil (100 mg/L), novobiocin $(32 \mathrm{mg} / \mathrm{L})$, and trimethoprim $(64 \mathrm{mg} / \mathrm{L})$ as a selective supplement. All antimicrobial substances were obtained as laboratory standard powders from Sigma (St. Louis, MO). After $48 \mathrm{~h}$ of incubation at $28 \pm$ $1^{\circ} \mathrm{C}$ under microaerobic conditions, a $10-\mu \mathrm{L}$ aliquot of the enrichment broth was streaked onto selective agar plates prepared by suspending $24 \mathrm{~g}$ of Arcobacter broth (Oxoid Ltd.) and $12 \mathrm{~g}$ of Agar Technical No. 3 (Oxoid Ltd.) and supplemented with selective antibiotic mix supplement as described above. The plates were incubated microaerobically at $28 \pm 1^{\circ} \mathrm{C}$ for $48 \mathrm{~h}$. The microaerobic conditions were created by evacuating $80 \%$ of the normal atmosphere and introducing a gas mixture of $8 \% \mathrm{CO}_{2}, 8 \% \mathrm{H}_{2}$, and $84 \% \mathrm{~N}_{2}$ into the jar. Up to 10 colonies, if present, suspected to be Arcobacter spp. were picked from each plate, subcultured, and subjected to presumptive identification using tests that included Gram stain and growth under aerobic conditions and cellular morphology.

For identification of the presumptive Arcobacter colonies, the isolates were subjected to DNA extraction using the REDExtract-N-Amp Tissue PCR Kit (Sigma), and identified by the multiplex PCR described by Douidah et al. (2010); isolates identified as A. butzleri were confirmed by the multiplex PCR described by Houf et al. (2000). Two reference strains, A. butzleri DSM $8739^{\mathrm{T}}$ and $A$. cryaerophilus DSM $7289^{\mathrm{T}}$ (Leibniz Institute DSMZ, Braunschweig, Germany), were used as controls: they were grown separately on nutrient agar supplemented with $5 \%$ laked horse blood (Oxoid Ltd.) incubated microaerobically at $30^{\circ} \mathrm{C}$ for $48 \mathrm{~h}$. 
Table 2. Samples collected in the 3 dairy plants investigated and number and description of positive samples

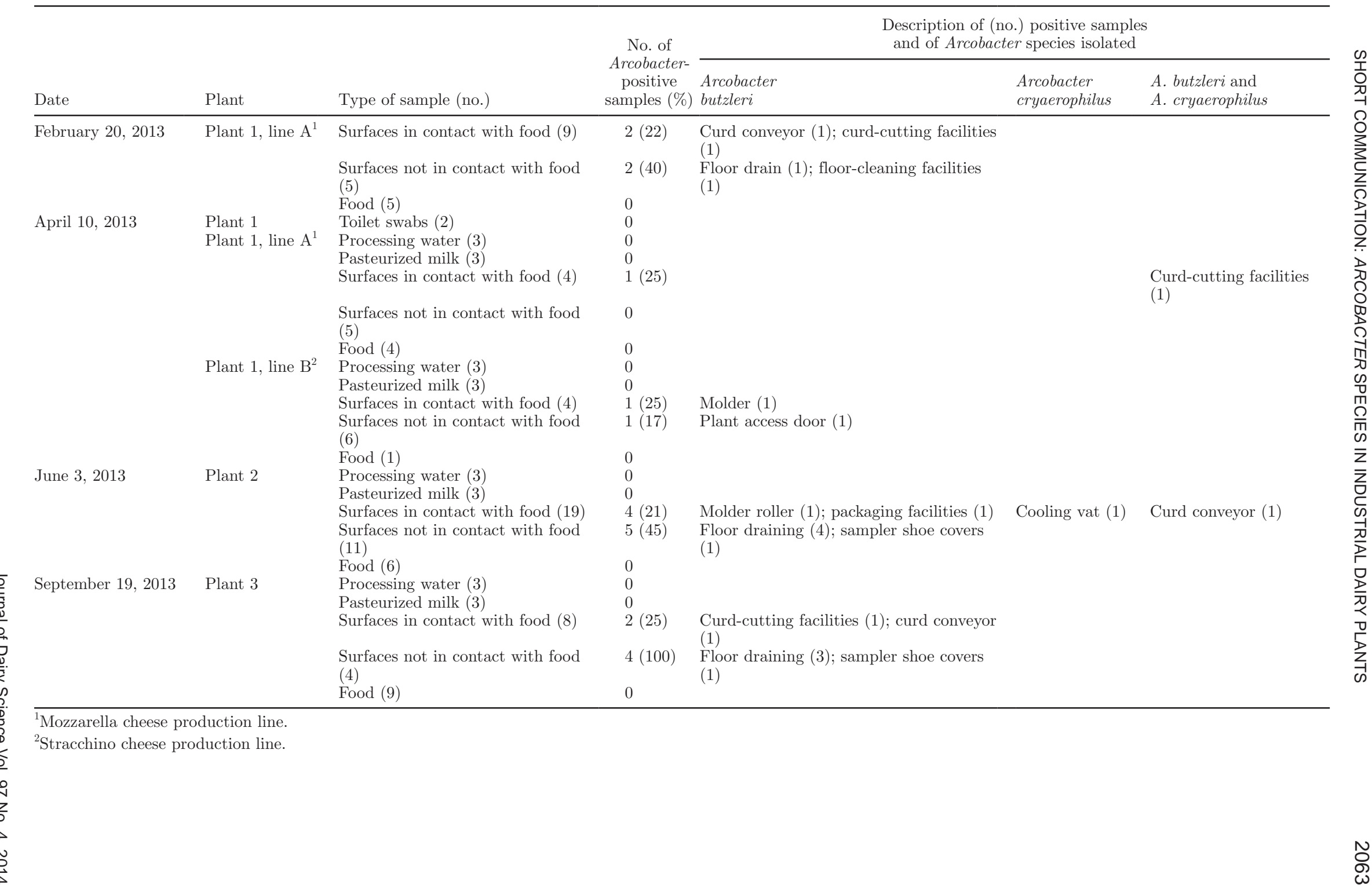


Arcobacter spp. were isolated from 22 of 75 environmental samples (29.3\%); namely, Arcobacter spp. were isolated from 12 of 31 surfaces not in contact with food $(38.7 \%)$ and from 10 of 44 surfaces in contact with food (22.7\%). All food (Mozzarella cheese, conditioning liquid, Stracchino cheese, and Ricotta cheese), processing water, and pasteurized milk samples were negative for Arcobacter spp. A total of 135 isolates were obtained; of these 129 and 6 were identified, respectively, as $A$. butzleri and A. cryaerophilus. The number and source of positive samples for each investigated dairy plant are detailed in Table 2 .

Arcobacter spp. were isolated in all the cheese factories investigated and in all the samplings performed: $A$. butzleri was the most prevalent species isolated, namely 21 of 22 positive samples, whereas A. cryaerophilus was isolated only in 3 of the overall 22 positive samples, in agreement with previous studies performed in poultry and cheese-processing plants (Ferreira et al., 2013; Giacometti et al., 2013a) and also in milk (Scullion et al., 2006; Shah et al., 2012; Serraino et al., 2013). In addition, the fact that $A$. butzleri displayed a longer survival time and better growth ability than $A$. cryaerophilus in milk (Giacometti et al., 2014), was more resistant in several substrates (D'Sa and Harrison, 2005; Van Driessche and Houf, 2008), and that the enrichment step reduces the diversity of Arcobacter species by favoring the faster-growing species (Houf et al., 2002), could account for the more frequent isolation of $A$. butzleri than other species in both milk and in environmental samples.

Both A. butzleri and A. cryaerophilus were isolated from surfaces in contact with food before and during manufacturing in all the plants investigated and in all the samplings performed. This demonstrates that Arcobacter spp. are not or are only partially affected by routine sanitizing procedures in the industrial dairy plants studied, as previously demonstrated in poultry slaughterhouses (Houf et al., 2002, 2003; Ferreira et al., 2013), a spinach processing plant (Hausdorf et al. 2013), and an artisanal dairy plant (Giacometti et al., 2013a). A recent study reported that $A$. butzleri isolates were able to tolerate sodium hypochlorite concentrations close to working solutions used for sanitizing in food processing plants and, hence, may survive the disinfection process (Rasmussen et al., 2013). The recurrent finding of Arcobacter spp. on sanitized surfaces highlights a crucial issue for industrial plants and calls for further studies to investigate their ability to survive sanitizing procedures.

In this study, we were not able to determine the primary source of contamination because we excluded pasteurized milk and water contamination, different from a previous study (Giacometti et al., 2013a), which identified raw milk used for cheesemaking as one of the prevalent sources of plant contamination.

To ensure safe food for consumers, food producers have a strong focus on hygiene during production. Cleaning and disinfection are the most common means of obtaining safe production environments and the presence of arcobacters, mainly A. butzleri, observed in the studied industrial dairy plants merits attention by food safety managers. On one hand, the observed Arcobacter spp. isolation from floors and floor drains, where they probably encounter favorable conditions for survival, may represent a source of spread through the plant by footwear and trolleys (as demonstrated by the isolation twice of $A$. butzleri from the shoe covers used by the sampler). On the other hand, the observed Arcobacter spp. isolation on food processing surfaces may represent a source of postprocessing food contamination.

In conclusion, the efficacy of sanitizing procedures against Arcobacter spp. should be evaluated and further studies are needed to establish whether certain Arcobacter strains persist for long periods of time in industrial dairy plants and whether they can survive in different types of cheese in cases of postprocessing contamination.

\section{REFERENCES}

Collado, L., and M. J. Figueras. 2011. Taxonomy, epidemiology, and clinical relevance of the genus Arcobacter. Clin. Microbiol. Rev. 24:174-192.

D'Sa, E. M., and M. A. Harrison. 2005. Effect of pH, NaCl content, and temperature on growth and survival of Arcobacter spp. J. Food Prot. 68:18-25.

Douidah, L., L. De Zutter, P. Vandamme, and K. Houf. 2010. Identification of five human and mammal associated Arcobacter species by a novel multiplex-PCR assay. J. Microbiol. Methods 80:281-286.

Ertas, N., Y. Dogruer, Z. Gonulalan, A. Guner, and I. Ulger. 2010 Prevalence of Arcobacter species in drinking water, spring water, and raw milk as determined by multiplex PCR. J. Food Prot. 73:2099-2102.

Ferreira, S., M. J. Fraqueza, J. A. Queiroz, F. C. Domingues, and M. Oleastro. 2013. Genetic diversity, antibiotic resistance and biofilmforming ability of Arcobacter butzleri isolated from poultry and environment from a Portuguese slaughterhouse. Int. J. Food Microbiol. 162:82-88.

Giacometti, F., A. Lucchi, G. Manfreda, D. Florio, R. G. Zanoni, and A. Serraino. 2013a. Occurrence and genetic diversity of Arcobacter butzleri in an artisanal dairy plant in Italy. Appl. Environ. Microbiol. 79:6665-6669.

Giacometti, F., A. Serraino, G. Marchetti, E. Bonerba, D. Florio, E. Bonfante, R. G. Zanoni, and R. Rosmini. 2013b. Isolation of Arcobacter butzleri in environmental and food samples collected in industrial and artisanal dairy plants. Ital. J. Food Saf. 2:121-123.

Giacometti, F., A. Serraino, F. Pasquali, A. De Cesare, E. Bonerba, and R. Rosmini. 2014. Behavior of Arcobacter butzleri and Arcobacter cryaerophilus in ultrahigh-temperature, pasteurized, and raw cow's milk under different temperature conditions. Foodborne Pathog. Dis. 11:15-20. http://dx.doi.org/10.1089/fpd.2013.1597.

González, A., and M. A. Ferrús. 2011. Study of Arcobacter spp. contamination in fresh lettuces detected by different cultural and molecular methods. Int. J. Food Microbiol. 145:311-314.

Hausdorf, L., M. Neumann, I. Bergmann, K. Sobiella, K. Mundt, A. Fröhling, O. Schlüter, and M. Klocke. 2013. Occurrence and ge- 
netic diversity of Arcobacter spp. in a spinach-processing plant and evaluation of two Arcobacter-specific quantitative PCR assays. Syst. Appl. Microbiol. 36:235-243.

Ho, H. T. K., L. J. A. Lipman, and W. Gaastra. 2006. Arcobacter, what is known and unknown about a potential foodborne zoonotic agent! Vet. Microbiol. 115:1-13.

Houf, K., L. De Zutter, J. Van Hoof, and P. Vandamme. 2002. Occurrence and distribution of Arcobacter species in poultry processing. J. Food Prot. 65:1233-1239.

Houf, K., L. De Zutter, J. Van Hoof, B. Verbeke, and P. Vandamme. 2003. Molecular characterization of Arcobacter isolates collected in a poultry slaughterhouse. J. Food Prot. 66:364-369.

Houf, K., L. A. Devriese, L. De Zutter, J. Van Hoof, and P. Vandamme. 2001. Development of a new protocol for the isolation and quantification of Arcobacter species from poultry products. Int. J. Food Microbiol. 71:189-196.

Houf, K., A. Tutenel, L. De Zutter, J. V. Hoof, and P. Vandamme. 2000. Development of a multiplex PCR assay for the simultaneous detection and identification of Arcobacter butzleri, Arcobacter cryaerophilus and Arcobacter skirrowii. FEMS Microbiol. Lett. 193:89-94.

Piva, S., A. Serraino, D. Florio, F. Giacometti, F. Pasquali, G. Manfreda, and R. G. Zanoni. 2013. Isolation of Arcobacter species in water buffaloes (Bubalus bubalis). Foodborne Pathog. Dis. 10:475-477.
Rasmussen, L. H., J. Kjeldgaard, J. P. Christensen, and H. Ingmer. 2013. Multilocus sequence typing and biocide tolerance of Arcobacter butzleri from Danish broiler carcasses. BMC Res. Notes $6: 322$.

Scullion, R., C. S. Harrington, and R. H. Madden. 2006. Prevalence of Arcobacter spp. in raw milk and retail raw meats in Northern Ireland. J. Food Prot. 69:1986-1990.

Serraino, A., D. Florio, F. Giacometti, S. Piva, D. Mion, and R. G. Zanoni. 2013. Presence of Campylobacter and Arcobacter species in in-line milk filters of farms authorized to produce and sell raw milk and of a water buffalo dairy farm in Italy. J. Dairy Sci. 96:2801-2807.

Shah, A. H., A. A. Saleha, M. Murugaiyah, Z. Zunita, and A. A. Memon. 2012. Prevalence and distribution of Arcobacter spp. in raw milk and retail raw beef. J. Food Prot. 75:1474-1478.

Shah, A. H., A. A. Saleha, Z. Zunita, M. Murugaiyah, A. B. Aliyu, and N. Jafri. 2013. Prevalence, distribution and antibiotic resistance of emergent Arcobacter spp. from clinically healthy cattle and goats. Transbound. Emerg. Dis. 60:9-16.

Van Driessche, E., and K. Houf. 2008. Survival capacity in water of $A r$ cobacter species under different temperature conditions. J. Appl. Microbiol. 105:443-451. 